\title{
Combined expression of aldehyde dehydrogenase 1A1 and $\beta$-catenin is associated with lymph node metastasis and poor survival in breast cancer patients following cyclophosphamide treatment
}

\author{
MINGLI SUN ${ }^{1}$, HAISHAN ZHAO $^{1}$, QINGHUAN XIAO ${ }^{2}$, ZHAOJIN YU $^{1}$, ZHIGUO SONG $^{1}$, \\ WEIFAN YAO ${ }^{1}$, HONGTAO TANG $^{1}$, SHU GUAN ${ }^{3}$, FENG JIN $^{3}$ and MINJIE WEI ${ }^{1}$ \\ Departments of ${ }^{1}$ Pharmacology and ${ }^{2}$ Ion Channel Pharmacology, School of Pharmacy, China Medical University, \\ Shenyang, Liaoning 110122; ${ }^{3}$ Department of Breast Surgery, The First Hospital of China Medical University, \\ Shenyang, Liaoning 110001, P.R. China
}

Received June 29, 2015; Accepted August 7, 2015

DOI: 10.3892/or.2015.4273

\begin{abstract}
This study investigated the expression of ALDH1A1 and $\beta$-catenin in breast cancer patients, and analyzed the correlation of their combined expression with clinicopathological features, chemotherapeutic responses, and prognosis of breast cancer patients. In total 276 human breast cancer tissues and 80 benign hyperplasia tissues were included. The expression of ALDH1A1 and $\beta$-catenin was examined using tissue microarray-based immunohistochemistry. ROC curve analysis was performed to determine an optimal cut-off score for the expression of ALDH1A1 and $\beta$-catenin, based on the survival status of breast cancer patients. Survival probabilities were estimated by the Kaplan-Meier method. ALDH1A1 expression was higher, but $\beta$-catenin showed no significant difference in breast cancer samples compared to controls. Compared with the membrane expression of $\beta$-catenin $[\beta$-catenin $(m)]$, the cytoplasmic expression of $\beta$-catenin $[\beta$-catenin(c)] occurred significantly more frequently in breast cancer with the high expression of ALDH1A1 [ALDH1A1(high)] than in breast cancer with the low expression of ALDH1A1 [ALDH1A1(low)] $(\mathrm{P}=0.014)$. The expression level of ALDH1A was significantly higher in $\beta$-catenin(c) breast cancer than in $\beta$-catenin $(\mathrm{m})$ breast cancer $(\mathrm{P}=0.020)$. ALDH1A1(high) expression or $\beta$-catenin(c) expression alone was associated with lymph node metastasis, and worse clinical outcome in breast cancer patients, especially in patients receiving cyclophosphamide treatment.
\end{abstract}

Correspondence to: Professor Minjie Wei, Department of Pharmacology, School of Pharmacy, China Medical University, 77 Puhe Road, Shenyang North New Area, Shenyang, Liaoning 110122, P.R. China

E-mail: weiminjiecmu@163.com

Key words: $\beta$-catenin, survival, breast cancer, cyclophosphamide treatment, aldehyde dehydrogenase $1 \mathrm{~A} 1$
Combined expression of ALDH1A1(high) and $\beta$-catenin(c) was associated with lymph node metastasis, poor outcome, and resistance to cyclophosphamide treatment. $\beta$-catenin may regulate ALDH1A1 expression in a subtype of breast cancer with ALDH1A1(high) and $\beta$-catenin(c) expression. ALDH1A1(high) and $\beta$-catenin(c) expression may be used as a biomarker for predicting poor prognosis in breast cancer following cyclophosphamide treatment.

\section{Introduction}

Breast cancer is one of the leading causes of cancer-related deaths in women. Breast cancer is a group of heterogeneous diseases with various responses to chemotherapy (1). Cancer stem cells (CSCs) are the tumor-initiating cells that are believed to be responsible for breast cancer recurrence, metastasis, and drug resistance $(2,3)$. CSCs express specific biomarkers such as CD44, aldehyde dehydrogenase 1A1 (ALDH1A1), and $\beta$-catenin (4-6). Expression of these CSC markers has prognostic values for the recurrence, metastasis, and therapeutic resistance in various tumors such as lung, hepatocellular, and breast cancer (7-9).

ALDH1, a member of a family of $\mathrm{NAD}(\mathrm{P})^{+}$-dependent enzymes that detoxify a wide variety of aldehydes, have been found to be associated with poor prognosis in breast cancer (10). ALDH1A1 has been extensively studied, and is found to be associated with tumor progression and poor prognosis of breast cancer patients $(11,12)$. ALDH1A1 plays an important role in the proliferation of breast CSCs and resistance to chemotherapy, particularly cyclophosphamide $(13,14)$. However, the signaling pathways that regulate the expression of ALDH1A1 in breast cancer remain unclear.

Recently, Condello et al have reported that $\beta$-catenin upregulates the expression of ALDH1A1, and $\beta$-catenin-regulated ALDH1A1 contributes to the maintenance of ovarian cancer spheroids (15). $\beta$-catenin is located in the intracellular side of the cytoplasmic membrane, and plays an important role in cell-to-cell adhesion by linking the cytoskeleton and 
cadherin (16). It is well known that $\beta$-catenin is involved in the Wnt signaling pathway that plays an active role in CSCs and carcinogenesis of various tumors such as breast cancer $(17,18)$ and ovarian cancer (19). Activation of Wnt signaling leads to translocation of $\beta$-catenin from the membrane to the cytoplasm and nucleus, where it promotes transcription of many genes that are associated with cancer growth, invasion, progression, and invasion (20). However, it remains unclear whether $\beta$-catenin regulates the expression of ALDH1A1 in breast cancer patients.

In the present study, we investigated the expression of ALDH1A1 and $\beta$-catenin in 276 breast cancer patients. We studied the association between the expression of ALDH1A1 and $\beta$-catenin in breast cancer patients, and analyzed the correlation of their combined expression with clinicopathological features, chemotherapeutic responses, and prognosis of breast cancer patients.

\section{Materials and methods}

Patients and tissue samples. The Medical Ethics Committee of China Medical University approved this study. Due to the retrospective nature of the study, the Medical Ethics Committee waived the need of written informed consents by the patients.

This study included human breast tissues from 276 female patients with breast cancer, who underwent surgery at the First Affiliated Hospital of China Medical University between 2004 and 2008. Breast cancer was diagnosed based on pathological staining. The patient age, menopausal status, tumor type, tumor size, and lymph node metastasis were retrospectively obtained from medical records. Of the 276 breast cancer patients, 220 had invasive ductal carcinoma, 30 had invasive lobular carcinoma, and 26 had other types of tumors including mucinous, medullary, and cribriform carcinoma. The histological grade of the cancer was determined according to the World Health Organization grading system. The stage of the cancer was evaluated according to the TNM staging system.

No patients underwent radiation therapy and chemotherapy before surgery. After surgery, 165 patients received adjuvant chemotherapy, and the remaining 11 patients did not receive chemotherapy. All chemotherapy regimens contained cyclophosphamide, including CMF (cyclophosphamide + methotrexate + fluorouracil, $\mathrm{n}=65)$, AC (adriamycin + cyclophosphamide, $n=32$ ), CEF (cyclophosphamide + epimbicin + fluorouracil, $n=32$ ), CE (cyclophosphamide + epimbicin, $\mathrm{n}=20$ ), and CAF (cyclophosphamide + adriamycin + fluorouracil, $\mathrm{n}=18)$.

Tissue microarray (TMA). Paraffinized donor blocks were stained with hematoxylin and eosin, and representative breast cancer samples were selected by reviewing the hematoxylin and eosin-stained blocks. Tissue cores with a diameter of $1.5 \mathrm{~mm}$ were extracted from each donor block, and precisely arrayed into a new paraffin recipient block with a maximum of 200 cores, using the Organization Microarrayer (Pathology Devices, USA).

Immunohistochemistry.Immunohistochemistry wasperformed as previously described (21). Briefly, sections (4- $\mu \mathrm{m}$ thick) were obtained from formalin-fixed and paraffin-embedded TMA recipient blocks, and mounted on poly-L-lysine-coated glass slides. Sections were deparaffinized with xylene, rehydrated in a graded alcohol series, and heated in a microwave oven to retrieve antigen. Sections were incubated with primary antibodies against ALDH1A1 (mouse anti-human monoclonal antibodies, 1:100 dilution; Abcam, UK) and $\beta$-catenin (mouse anti-human monoclonal antibodies, 1:200 dilution; Cell Signaling Technology, USA) overnight at $4^{\circ} \mathrm{C}$, followed by incubation with biotinylated secondary antibodies for $30 \mathrm{~min}$ at $37^{\circ} \mathrm{C}$. Sections were then incubated with streptavidin horseradish peroxidase for additional $30 \mathrm{~min}$ (LSAB kit; Dako, Glostrup, Denmark), and stained with 3,3-diaminobenzidine (DAB). Sections were counterstained with hematoxylin, dehydrated, and mounted. Negative control sections were treated in the same conditions, except no primary antibodies.

Evaluation of immunohistochemistry. The immunostaining was examined under a light microscope, and was evaluated by two pathologists blinded to the experimental conditions. The immunoreactivity intensity was scored as follows: 0 for negative staining, 1 for weak positive staining, 2 for moderate positive staining, and 3 for strong positive staining. The percentage of stained cells was scored $0-100 \%$. The final immunoreactive score was calculated by multiplying the intensity score with the score for the percentage of positively stained cells, ranging from 0 to $300 \%$. The scores were assigned by using 5\% increments $(0,5,10$, and $300 \%)$ as previously reported $(22,23)$. The final immunoreactive scores were used to determine the cut-off value for discriminating tumors with the high expression of ALDH1A1 or $\beta$-catenin from those with low expression, using receiver operating characteristic (ROC) curves. To discriminate tumors with the cytoplasmic expression of $\beta$-catenin from tumor with the membrane expression of $\beta$-catenin, the percentage of $\beta$-catenin membrane expression for each sample was used to determine the cut-off value, using ROC curves. The percentage of $\beta$-catenin membrane expression was calculated as follows: $\beta$-catenin membrane expression $\%=$ The number of cells with membrane expression/(the number of cells with membrane expression + the number of cells with cytoplasmic expression) $x \quad 100 \%$. The sensitivity and specificity for the survival status (alive or dead) of breast cancer patients was plotted to generate ROC curves.

Statistical analysis. Statistical analyses were performed using SPSS 16.0 (SPSS, Inc., Chicago, IL, USA). For data with normal distribution, Student's t-test was used to compare the difference in the means. For data with unequal variance, Wilcoxon rank sum tests or Kruskal-Wallis tests were used to compare the difference. The association between the expression of ALDH1A1 and $\beta$-catenin and clinicopathological characteristics of breast cancer patients was evaluated using the Pearson's Chi-square test or Fisher's exact probability test. The Pearson's rank correlation analysis was applied to assess the association between the expression of ALDH1A1 and $\beta$-catenin. The Kaplan-Meier curves were plotted to demonstrate the survival difference, and the survival probabilities were assessed by a log-rank test. To assess the association of potential confounding variables with the prognosis, overall survival (OS), or disease-free survival (DFS), univariate and 
Table I. Clinicopathological characteristics of breast cancer patients.

\begin{tabular}{|c|c|c|}
\hline & \multicolumn{2}{|c|}{ Cases } \\
\hline & $\mathrm{n}$ & $\%$ \\
\hline Age (years) & 276 & \\
\hline$\leq 50$ & 149 & 53.9 \\
\hline$>50$ & 127 & 46.1 \\
\hline Menopausal status & 266 & \\
\hline Pre-menopause & 150 & 56.4 \\
\hline Post-menopause & 116 & 43.6 \\
\hline Tumor size (cm) & 229 & \\
\hline$\leq 2.0$ & 142 & 62.0 \\
\hline$>2.0$ & 87 & 38.0 \\
\hline Tumor type & 276 & \\
\hline Ductal & 220 & 79.7 \\
\hline Lobular & 30 & 10.9 \\
\hline Others & 26 & 9.4 \\
\hline Histological grade & 267 & \\
\hline I & 136 & 50.9 \\
\hline II-III & 131 & 49.1 \\
\hline TNM stage & 198 & \\
\hline I & 46 & 23.4 \\
\hline II & 122 & 61.9 \\
\hline III & 29 & 14.7 \\
\hline Lymph node metastasis & 265 & \\
\hline No & 175 & 65.8 \\
\hline Yes & 91 & 34.2 \\
\hline
\end{tabular}

multivariate Cox proportional hazards regression models were used. The OS was defined as the time between the day of diagnosis and the disease-related death, or last known follow-up. The DFS was calculated as the time between the day of diagnosis and the occurrence of local recurrence or distant metastasis. Probability values $\leq 0.05$ were considered statistically significant.

\section{Results}

Clinicopathological characteristics. Table I summarizes clinicopathological characteristics of 276 breast cancer patients. The average age of breast cancer patients was $50.1 \pm 7.9$ years (range, 31-82 years). Of the 276 patients, the age, menopausal status, tumor size, tumor type, histological grade, TNM stage, and lymph node metastasis were recorded in $276,266,229,276,267,197$, and 266 patients, respectively. The majority of these patients had a tumor with invasive ductal carcinoma $(79.7 \%)$, and $<2 \mathrm{~cm}$ in size $(62.0 \%)$. Of the 267 patients with known histological grade, $136(50.9 \%)$ patients had grade I breast cancer, and 131 (49.1\%) patients had grade II-II breast cancer. The stage of the cancer was evaluated in 197 patients, including 46 patients with stage I breast cancer, 122 patients with stage II breast cancer, and 29 patients with stage III breast cancer. Lymph node metastasis occurred in $91(34.2 \%)$ of 266 patients.

Follow-up information was available for 168 breast cancer patients. During the follow-up period of 9-118 months, relapses occurred in 62 cases and cancer-associated deaths were found in 48 cases. The 5-years survival rate was $84.5 \%$. The mean OS and DFS were 98.5 and 91.5 months, respectively.

Expression of ALDHIAI and $\beta$-catenin. We studied the expression of ALDH1A1 and $\beta$-catenin in 276 samples from breast cancer patients and 80 control samples from patients with benign hyperplasia, using immunohistochemistry (Fig. 1). ALDH1A1-immunopositive staining was mainly observed in the cytoplasm in both breast cancer and control samples. ALDH1A1-immunopositive staining was observed

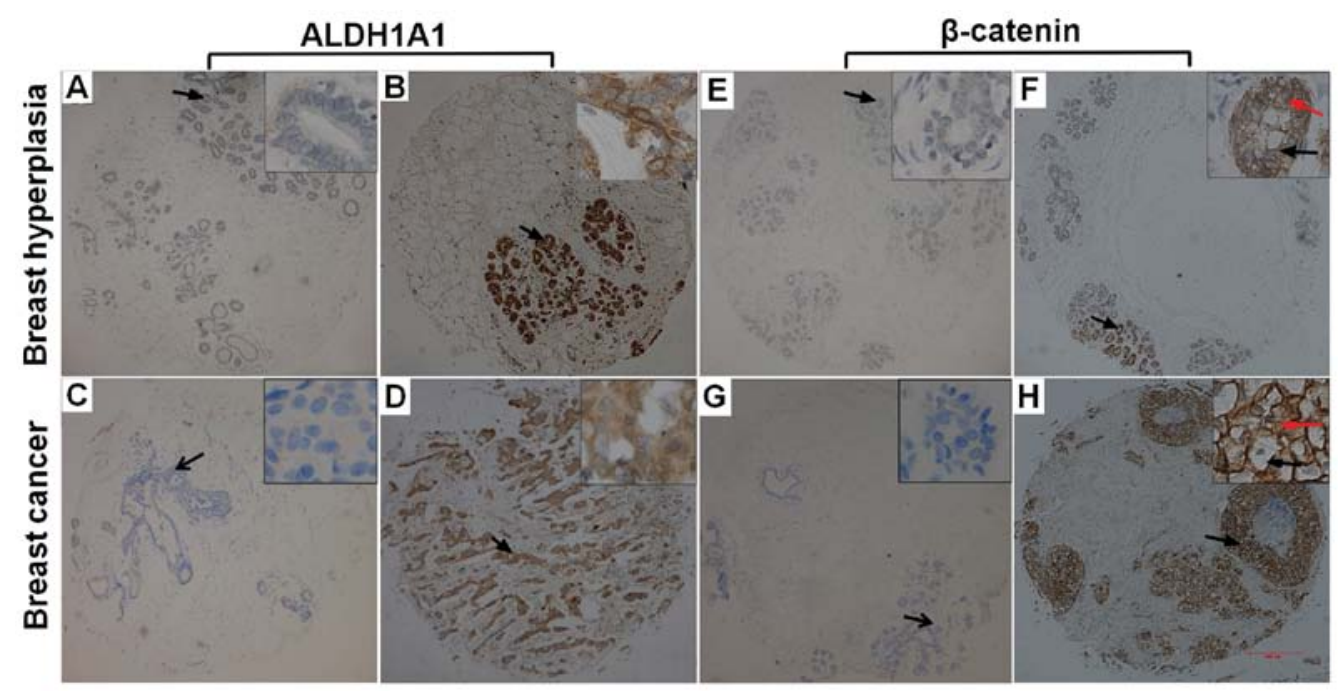

Figure 1. Representative micrographs showing negative (A, C, E and G) and positive (B, D, F and H) immunohistochemical staining of ALDH1A1 (A-D) and $\beta$-catenin $(\mathrm{E}-\mathrm{H})$ in breast hyperplasia and breast cancer. Magnification, $\mathrm{x} 40$. Arrows indicate the magnified regions in the insert (x400). Black arrow in the magnified regions indicate $\beta$-catenin membrane expression and red arrow indicate $\beta$-catenin cytoplasmic expression. Scale bar, $200 \mu \mathrm{m}$. ALDH1A1, aldehyde dehydrogenase $1 \mathrm{~A} 1$. 

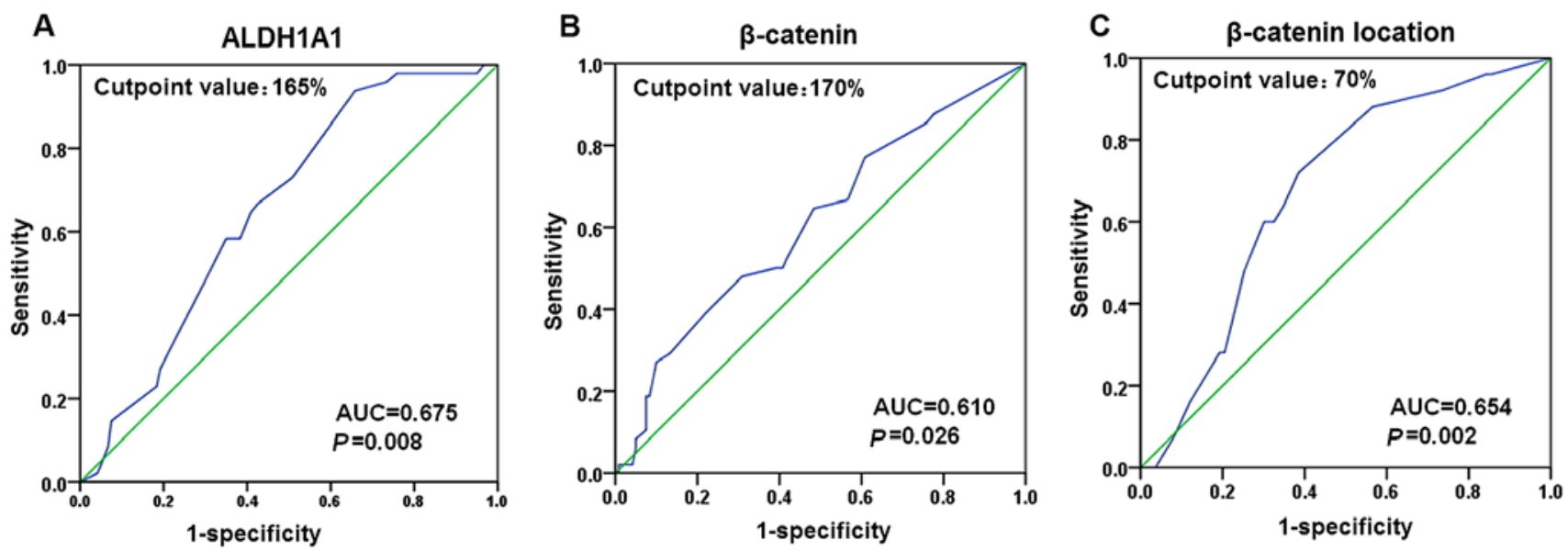

Figure 2. Receiver operating characteristic curves were used to determine the cut-off score for the expression of ALDH1A1 (A) and $\beta$-catenin (B), and subcellular expression of $\beta$-catenin (C) based on the survival status of breast cancer patients. The sensitivity and specificity for each outcome were plotted and the AUCs and p-value were indicated. ALDH1A1, aldehyde dehydrogenase 1A1; AUCs, areas under curve.

A
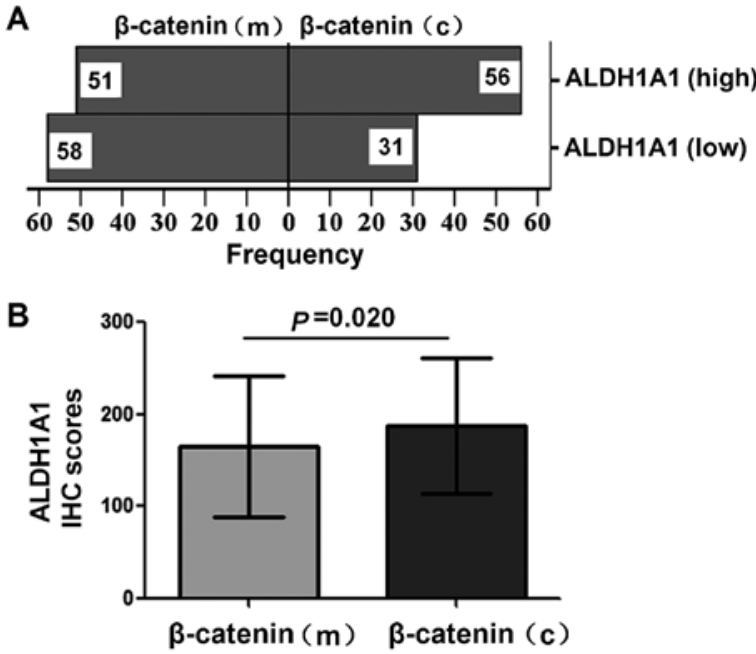

Figure 3. The association between the subcellular expression of and $\beta$-catenin and ALDH1A1 expression in breast cancer. (A) $\beta$-catenin(m) and $\beta$-catenin(c) expression in breast cancer with ALDH1A1(high) and ALDH1A1(low) expression. (B) ALDH1A1 IHC scores in breast cancer with $\beta$-catenin $(\mathrm{m})$ and $\beta$-catenin(c) expression. Data are presented as mean $\pm \mathrm{SD}$. $\mathrm{P}=0.029$. ALDH1A1, aldehyde dehydrogenase 1A1.

in 149 (54\%) of 276 breast cancer samples and $25(31.3 \%)$ of 80 control samples. ALDH1A1 immunoreactivity occurred significantly more frequently in breast cancer samples than in control samples $(\mathrm{P}<0.001)$. In contrast, $\beta$-catenin immunoreactivity was observed in the membrane and cytoplasm in both breast cancer samples and control samples. $\beta$-catenin immunoreactivity was observed in $246(89.1 \%)$ of 276 breast cancer samples and $72(90.0 \%)$ of 80 control samples. There was no significant difference of $\beta$-catenin-immunopositive staining between breast cancer samples and control samples $(\mathrm{P}=0.824)$.

Selection of the cut-off value for the expression of ALDHIAl and $\beta$-catenin. ROC curve analysis was performed to determine an optimal cut-off score for the expression of ALDH1A1 and $\beta$-catenin in breast cancer samples. Based on the survival status, a cut-off score of 165 and $170 \%$ were selected for the expression of ALDH1A1 and $\beta$-catenin, respectively (Fig. 2A and B). Tumors with immunohistological scores $\geq 165$ and $<165 \%$ were defined as tumors with 'high' [ALDH1A1(high)] and 'low' [ALDH1A1(low)] expression of ALDH1A1, respectively. Tumors with immunohistological scores $\geq 170$ and $<170 \%$ were defined as tumors with 'high' $[\beta$-catenin(high)] and 'low' $[\beta$-catenin(low)] expression of $\beta$-catenin, respectively. In total 149 tumors (54.0\%) exhibited ALDH1A1(high) expression, and 127 (46.0\%) tumors showed ALDH1A1(low) expression; 196 (71.0\%) tumors exhibited $\beta$-catenin(high) expression, and 80 (29.0\%) tumors showed $\beta$-catenin(low) expression.

We further performed ROC analysis to determine an optimal cut-off score for discriminating the membrane expression from the cytoplasmic expression of $\beta$-catenin in breast cancer with $\beta$-catenin(high) expression. Based on the survival status, a cut-off score of $70 \%$ was selected (Fig. 2C). Tumors with the membrane expression $\geq 70$ and $<70 \%$ were defined as tumors with $\beta$-catenin(m), and $\beta$-catenin (c) expression, respectively. Of the $196 \beta$-catenin(high) breast cancers, 109 (55.6\%) had $\beta$-catenin(m) expression, and 87 (44.4\%) had $\beta$-catenin(c) expression.

Association between the expression of ALDHIAl and $\beta$-catenin. Pearson's rank correlation analysis was used to analyze the association between the expression of ALDH1A1 and $\beta$-catenin in breast cancer. There was no significant correlation between the expression of ALDH1A1 and $\beta$-catenin in the breast cancer patients $(n=276)$. We then examined the subcellular expression levels of $\beta$-catenin in breast cancer with $\beta$-catenin(high) expression. In breast cancer with ALDH1A1(low) expression, $\beta$-catenin(m) expression was observed in $58(65.2 \%)$ of 89 cases, and $\beta$-catenin(c) expression was found in 31 (34.8\%) of 89 cases. In breast cancer with ALDH1A1(high) expression, $\beta$-catenin(m) expression was observed in $51(47.7 \%)$ of 107 cases, and $\beta$-catenin(c) expression was found in $56(52.3 \%)$ of 107 cases. $\beta$-catenin(c) expression occurred significantly more frequently in ALDH1A1(high) breast cancer than in ALDH1A1(low) breast cancer (Chi-square test, $\mathrm{P}=0.014$; Fig. 3A). Furthermore, the expression level of ALDH1A was significantly higher 
Table II. Association of $\beta$-catenin localization and ALDH1A1 expression with clinicopathological features of breast cancer.

\begin{tabular}{|c|c|c|c|c|c|c|}
\hline & \multicolumn{3}{|c|}{ ALDH1A 1} & \multicolumn{3}{|c|}{$\beta$-catenin localization } \\
\hline & $\operatorname{High}(\mathrm{n} / \%)$ & Low $(\mathrm{n} / \%)$ & P-value & $\begin{array}{c}\beta \text {-catenin }(\mathrm{c}) \\
(\mathrm{n} / \%)\end{array}$ & $\underset{(\mathrm{n} / \%)}{\beta \text {-catenin }(\mathrm{m})}$ & P-value \\
\hline Age at diagnosis (years) & & & 0.690 & & & 0.364 \\
\hline$\leq 50$ & $80 / 55.2$ & $65 / 44.8$ & & $42 / 36.2$ & $61 / 63.8$ & \\
\hline$>50$ & $67 / 52.8$ & $60 / 47.2$ & & $43 / 47.3$ & $48 / 52.7$ & \\
\hline Menopausal state & & & 0.736 & & & 0.908 \\
\hline Pre-menopause & $82 / 54.7$ & $68 / 45.3$ & & $47 / 43.5$ & $61 / 56.5$ & \\
\hline Post-menopause & $61 / 52.6$ & $55 / 47.4$ & & $35 / 42.7$ & $47 / 57.3$ & \\
\hline Tumor size (cm) & & & 0.729 & & & 0.622 \\
\hline$\leq 2.0$ & $75 / 52.8$ & $67 / 47.2$ & & $45 / 35.5$ & $58 / 64.5$ & \\
\hline$>2.0$ & $48 / 55.2$ & $39 / 44.8$ & & $30 / 47.6$ & $33 / 52.4$ & \\
\hline Tumor type & & & 0.065 & & & 0.102 \\
\hline Ductal & $109 / 49.5$ & $111 / 50.5$ & & $76 / 48.1$ & $82 / 51.9$ & \\
\hline Lobular & $20 / 66.7$ & $10 / 33.3$ & & $6 / 30.0$ & $14 / 70.0$ & \\
\hline Others & $18 / 78.2$ & $5 / 21.8$ & & $5 / 27.8$ & $13 / 72.2$ & \\
\hline Histological grade & & & 0.876 & & & 0.014 \\
\hline I & $75 / 55.1$ & $61 / 44.9$ & & $35 / 36.1$ & $62 / 63.9$ & \\
\hline II-III & $71 / 54.2$ & $60 / 45.8$ & & $49 / 53.8$ & $42 / 46.2$ & \\
\hline TNM stage & & & 0.267 & & & 0.139 \\
\hline I-II & $87 / 51.5$ & $82 / 48.5$ & & $48 / 40.0$ & $72 / 60.0$ & \\
\hline III & $17 / 63.0$ & $10 / 37.0$ & & $9 / 60.0$ & $6 / 40.0$ & \\
\hline Lymph node metastasis & & & 0.029 & & & 0.002 \\
\hline No & $85 / 48.6$ & $90 / 51.4$ & & $48 / 36.5$ & $84 / 63.5$ & \\
\hline Yes & $57 / 62.6$ & $34 / 37.4$ & & $36 / 60.0$ & $24 / 40.0$ & \\
\hline
\end{tabular}

P-value obtained from Pearson's Chi-square test or Fisher's exact test; ALDH1A1, aldehyde dehydrogenase 1A1.

Table III. Association of the combination of $\beta$-catenin location and ALDH1A1 with lymph node metastasis in breast cancer.

\begin{tabular}{|c|c|c|c|c|c|}
\hline \multirow[b]{2}{*}{ Features } & \multicolumn{2}{|c|}{ Node metastasis } & \multicolumn{3}{|c|}{ P-value ${ }^{b}$} \\
\hline & No $(\%)^{\mathrm{a}}$ & Yes $(\%)^{\mathrm{a}}$ & & & \\
\hline ALDH1A1(low) and $\beta$-catenin(m) & $47(82.5)$ & $10(17.5)$ & 0.003 & & \\
\hline ALDH1A1(low) and $\beta$-catenin(c) & $37(72.5)$ & $14(27.5)$ & $0.216^{\mathrm{c}}$ & & \\
\hline ALDH1A1(high) and $\beta$-catenin(m) & $21(70.0)$ & $9(30.0)$ & $0.181^{\mathrm{c}}$ & $0.806^{\mathrm{d}}$ & \\
\hline ALDH1A1(high) and $\beta$-catenin(c) & $27(50.0)$ & $27(50.0)$ & $<0.001^{\mathrm{c}}$ & $\mathbf{0 . 0 1 8}$ & $0.076^{\mathrm{e}}$ \\
\hline
\end{tabular}

in $\beta$-catenin(c) breast cancer than in $\beta$-catenin(m) breast cancer ( $\mathrm{P}=0.020$, Fig. 3B).

Association of the expression of ALDHIAl and $\beta$-catenin with clinicopathological characteristics of breast cancer patients. Table II summarizes the association between the expression of ALDH1A1 and $\beta$-catenin and the clinicopathological characteristics of breast cancer patients. The age, menopausal status, tumor type, tumor size, histological grade, and TNM stage were not significantly associated with the expression of ALDH1A1. ALDH1A1(high) expression was associated with lymph node metastasis $(\mathrm{P}=0.029)$. The age, menopausal status, tumor size, tumor type, and TNM stage were not significantly associated with the subcellular expression of $\beta$-catenin. $\beta$-catenin(c) expression was associated with grade II-III tumors $(\mathrm{P}=0.014)$ and lymph node metastasis $(\mathrm{P}=0.002)$. 

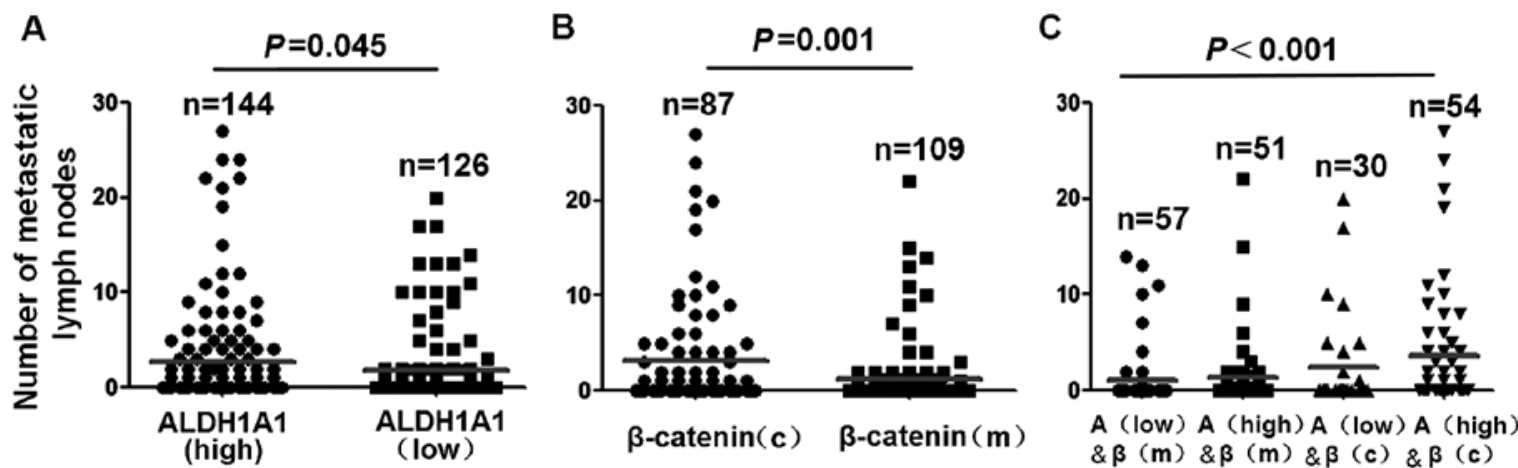

Figure 4. Breast cancer patients with ALDH1A1(high) and ALDH1A1(low) expression (A), $\beta$-catenin(c) and $\beta$-catenin(m) expression (B), and their combined expression (C). Red line denotes the median value. A and B, Wilcoxon rank sum test; $\mathrm{C}$, Kruskal-Wallis $\mathrm{H}$ test.

A

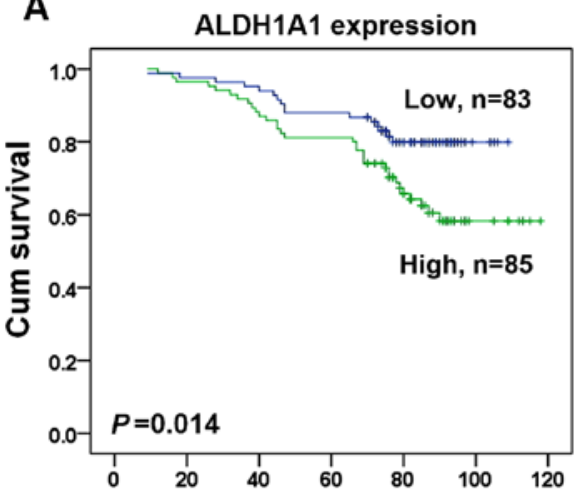

C

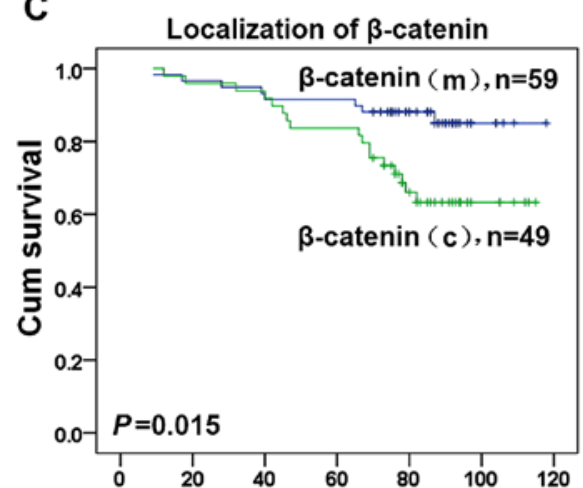

E

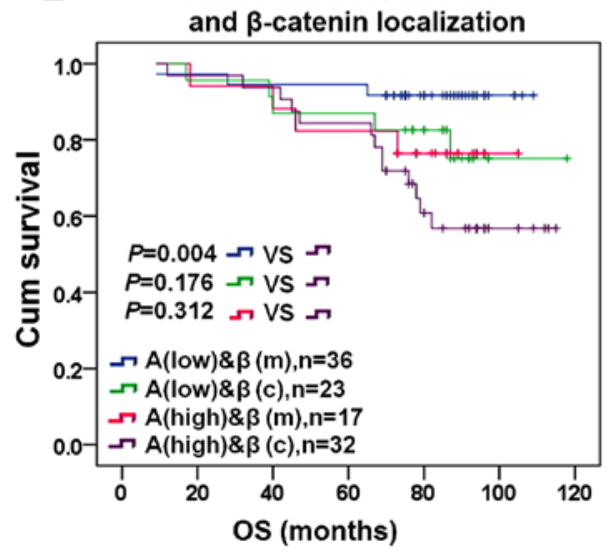

B

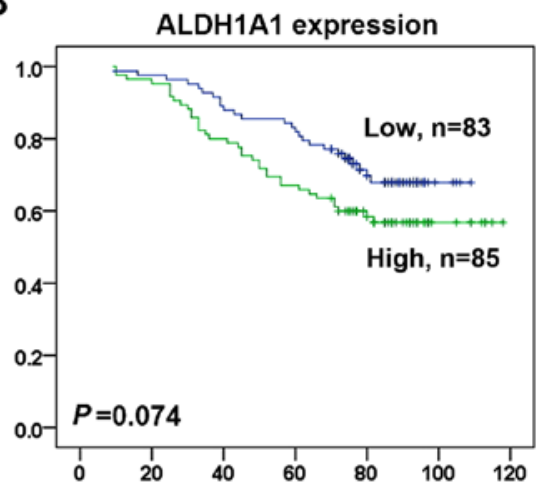

D

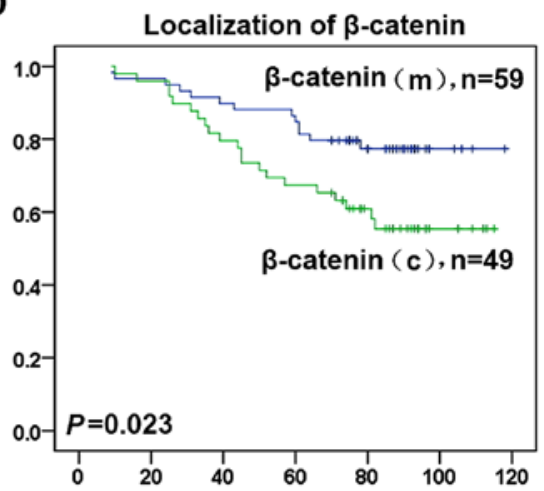

F Combination of ALDH1A1

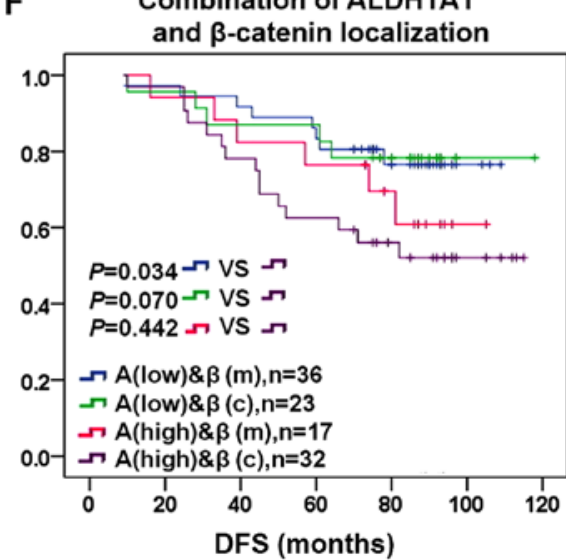

Figure 5. Kaplan-Meier survival analysis of ALDH1A1 and $\beta$-catenin expression in breast cancer patients. (A and B) Survival curves show the association between ALDH1A1 expression and OS (A) or DFS (B) in 168 breast cancer patients. (C and D) Survival curves show the association between the subcellular expression of $\beta$-catenin and OS (C) amd DFS (D) in 108 breast cancer patients. (E and F) Survival curves show the association between combined expression of ALDH1A1 and $\beta$-catenin and OS (E) or DFS (F) in 108 breast cancer patients. ALDH1A1, aldehyde dehydrogenase 1A1; OS, overall survival; DFS, disease-free survival. 
A

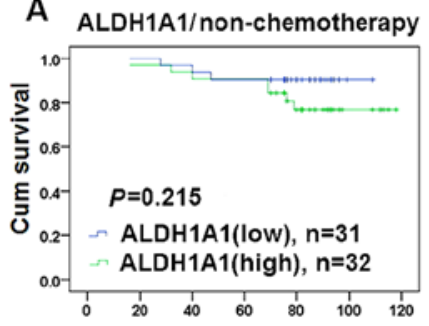

E $\quad \beta$-catenin localization

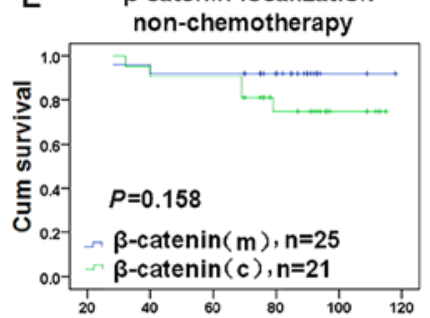

I ALDH1A1 and $\beta$-catenin
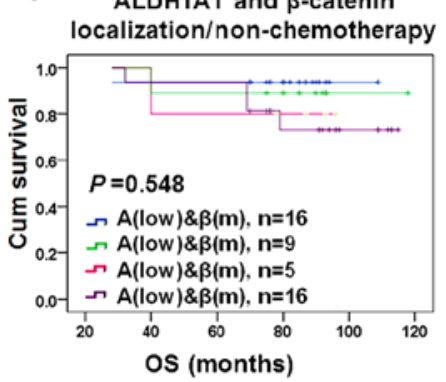

B

B ALDH1A1/non-chemotherapy
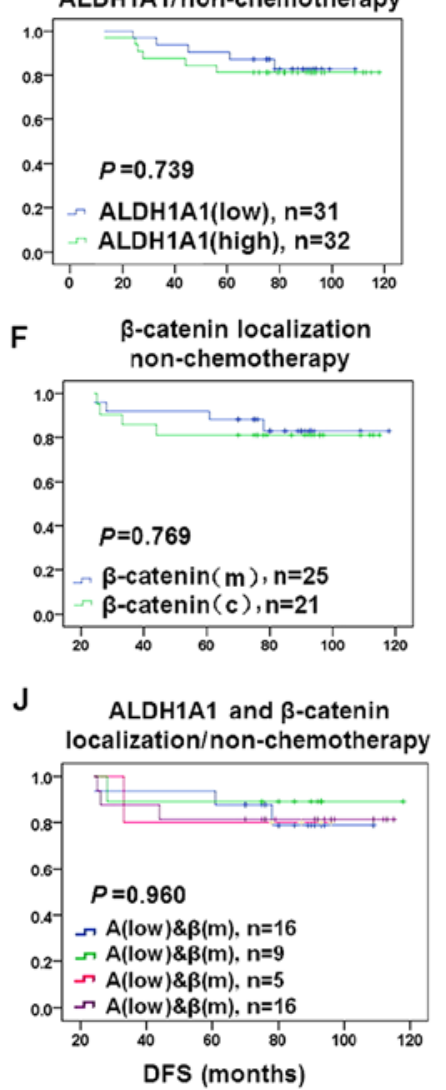

C

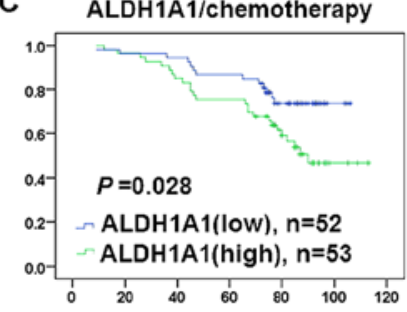

G

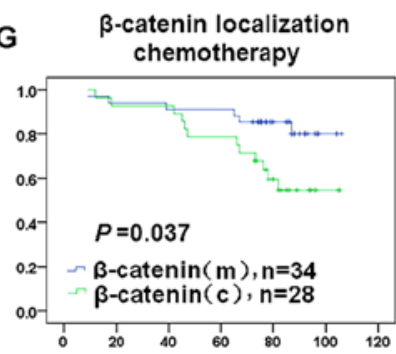

K ALDH1A1 and $\beta$-catenin

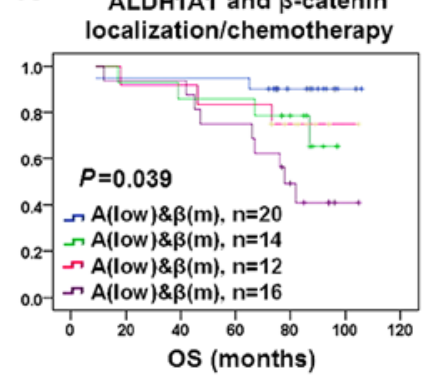

D ALDH1A1/chemotherapy

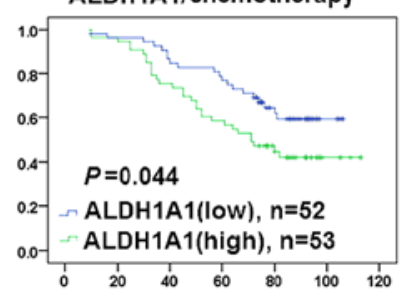

H $\quad \beta$-catenin localization chemotherapy

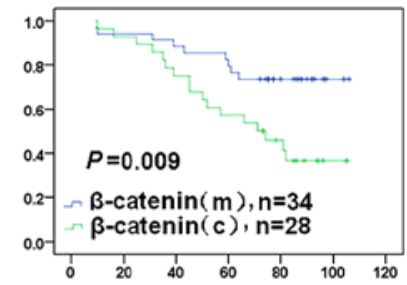

L

ALDH1A1 and $\beta$-catenin localization/chemotherapy

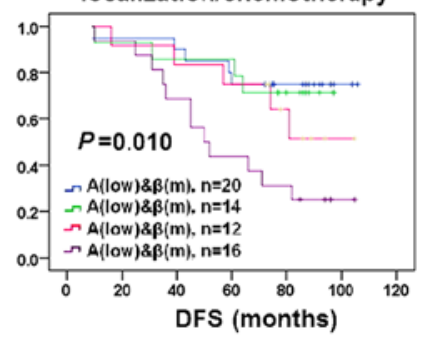

Figure 6. Kaplan-Meier survival analysis of ALDH1A1 and $\beta$-catenin expression in breast cancer patients with or without cyclophosphamide treatment. (A-D) Survival curves show the association between ALDH1A1 expression and OS (A and C) or RFS (B and D) in breast cancer patients receiving noncyclophosphamide (A and B) or cyclophosphamide (C and D) therapy. (E-H) Survival curves show the association between the subcellular expression of $\beta$-catenin and OS (E and G) or RFS ( $F$ and $\mathrm{H}$ ) in breast cancer patients receiving non-cyclophosphamide $(\mathrm{E}$ and $\mathrm{F})$ or cyclophosphamide $(\mathrm{G}$ and $\mathrm{H})$ therapy. (I-L) Survival curves show the association between the combined expression of ALDH1A1 and $\beta$-catenin and OS (I and L) or RFS (J and L) in breast cancer patients receiving non-cyclophosphamide (I and J) or cyclophosphamide (K and L) therapy. ALDH1A1, aldehyde dehydrogenase 1A1; OS, overall survival.

Table III summarizes the association of combined expression of ALDH1A1 and $\beta$-catenin with lymph node metastasis. Compared with tumors with ALDH1A1(low) and $\beta$-catenin(m) expression and ALDH1A1(low) and $\beta$-catenin(c) expression, tumors with ALDH1A1(high) and $\beta$-catenin(c) expression was associated with lymph node metastasis $(\mathrm{P}<0.05$, Table III). Compared with tumors with ALDH1 A1(high) and $\beta$-catenin(m) expression, tumors with ALDH1A1(high) and $\beta$-catenin(c) expression exhibited a tendency toward more lymph node metastasis ( $\mathrm{P}=0.076$, Table III).

We then examined the effect of ALDH1A1 and $\beta$-catenin expression on the number of metastatic lymph nodes in breast cancer. The number of metastatic lymph nodes was significantly higher in patients with ALDH1A1(high) expression compared with patients with ALDH1A1(low) expression ( $\mathrm{P}=0.045$, Fig. 4A). The number of metastatic lymph nodes was significantly higher in patients with $\beta$-catenin(c) expression compared with patients with $\beta$-catenin $(\mathrm{m})$ expression ( $\mathrm{P}=0.001$, Fig. 4B). Patients with ALDH1A1(high) and $\beta$-catenin(c) expression had significantly more metastatic lymph nodes compares with tumors with other combined expression of ALDH1A and $\beta$-catenin ( $\mathrm{P}<0.001$, Fig. 4C).

Association of the expression of ALDHIAl and $\beta$-catenin with the survival of breast cancer patients. The Kaplan-Meier anal- ysis and log-rank test were used to evaluate the association of the expression level of ALDH1A1 and $\beta$-catenin with the OS or DFS in breast cancer patients. ALDH1 A1(high) expression was associated with significantly shorter $\mathrm{OS}$ ( $\mathrm{P}=0.014$, Fig. 5A). Although ALDH1A1(high) expression was associated with a tendency toward shorter DFS in breast cancer patients, no significant difference was found ( $\mathrm{P}=0.074$, Fig. 5B). In addition, $\beta$-catenin(c) expression was associated with significantly shorter OS ( $\mathrm{P}=0.015$, Fig. 5C) and DFS ( $\mathrm{P}=0.023$, Fig. 5D). We further examined the association of the combined expression of ALDH1A1 and $\beta$-catenin with the OS or DFS in breast cancer patients. Compared with ALDH1A1(low) and $\beta$-catenin(m) expression, ALDH1A1(high) and $\beta$-catenin(c) expression was associated with shorter OS and DFS in breast cancer patients $(\mathrm{P}<0.05$, Fig. $5 \mathrm{E}$ and $\mathrm{F})$.

We examined the association of the expression of ALDH1A1 and $\beta$-catenin with the therapeutic response in breast cancer patients who received cyclophosphamide treatment. ALDH1A1 and $\beta$-catenin alone and their combined expression were not significantly associated with the OS and DFS in breast cancer patients with non-cyclophosphamide therapy (Fig. 6A, B, E, F, I and J). ALDH1 A1(high) expression was associated with shorter OS $(\mathrm{P}=0.004)$ and DFS $(\mathrm{P}=0.002)$ in patients with cyclophosphamide treatment (Fig. 6C and D). $\beta$-catenin(c) expression was associated with shorter OS 
Table IV. Univariate Cox regression analysis of the association between clinicopathological data and OS and DFS in breast cancer patients.

\begin{tabular}{|c|c|c|c|c|c|c|c|}
\hline & \multirow[b]{2}{*}{ Total no. } & \multicolumn{3}{|c|}{ OS } & \multicolumn{3}{|c|}{ DFS } \\
\hline & & $\begin{array}{l}\text { Events } \\
\mathrm{n}(\%)\end{array}$ & RR $(95 \%$ CI) & P-value & $\begin{array}{l}\text { Events } \\
\mathrm{n}(\%)\end{array}$ & RR $(95 \%$ CI) & P-value \\
\hline Age (years) & & & & 0.126 & & & 0.116 \\
\hline$\leq 50$ & 87 & $21(24.1)$ & 1 (reference) & & $27(31.0)$ & 1 (reference) & \\
\hline$>50$ & 79 & $27(34.2)$ & $1.561(0.883-2.763)$ & & $34(43.0)$ & $1.500(0.905-2.487)$ & \\
\hline Menopausal state & & & & 0.029 & & & 0.034 \\
\hline Pre-menopause & 87 & $19(21.8)$ & 1 (reference) & & $25(28.7)$ & 1 (reference) & \\
\hline Post-menopause & 75 & $28(37.3)$ & $1.911(1.067-3.424)$ & & $33(44.0)$ & $1.755(1.043-2.953)$ & \\
\hline Tumor size (cm) & & & & $<0.001$ & & & $<0.001$ \\
\hline$\leq 2.0$ & 76 & $11(14.5)$ & 1 (reference) & & $19(25.0)$ & 1 (reference) & \\
\hline$>2.0$ & 60 & $33(55.0)$ & $5.070(2.557-10.054)$ & & $38(63.3)$ & $3.546(2.036-6.173)$ & \\
\hline Tumor type & & & & 0.541 & & & 0.387 \\
\hline Ductal & 134 & $37(27.6)$ & 1 (reference) & & $47(35.1)$ & 1 (reference) & \\
\hline Lobular & 17 & $6(35.3)$ & $1.309(0.552-3.102)$ & & $7(41.2)$ & $1.178(0.813-1.706)$ & \\
\hline Histological grade & & & & $<0.001$ & & & $<0.001$ \\
\hline I & 79 & $6(7.6)$ & 1 (reference) & & $13(16.5)$ & 1 (reference) & \\
\hline II-III & 84 & $42(50.0)$ & 8.699 (3.687-20.524) & & $48(57.1)$ & $4.673(2.526-8.642)$ & \\
\hline TNM stage & & & & $<0.001$ & & & $<0.001$ \\
\hline I-II & 93 & $24(25.8)$ & 1 (reference) & & $31(33.3)$ & 1 (reference) & \\
\hline III & 20 & $15(75.0)$ & $5.039(2.625-9.673)$ & & $16(80.0)$ & $4.727(2.554-8.749)$ & \\
\hline $\begin{array}{l}\text { Lymph node } \\
\text { metastasis }\end{array}$ & & & & $<0.001$ & & & $<0.001$ \\
\hline No & 98 & $13(13.3)$ & 1 (reference) & & $18(18.4)$ & 1 (reference) & \\
\hline Yes & 62 & $32(51.6)$ & $4.997(2.617-9.542)$ & & $40(64.5)$ & $4.900(2.800-8.574)$ & \\
\hline $\begin{array}{l}\text { ALDH1A1 } \\
\text { expression }\end{array}$ & & & & 0.016 & & & 0.077 \\
\hline Low & 83 & $16(19.3)$ & 1 (reference) & & $25(30.1)$ & 1 (reference) & \\
\hline High & 85 & $32(37.6)$ & $2.085(1.144-3.800)$ & & $36(42.4)$ & $1.585(0.951-2.641)$ & \\
\hline $\begin{array}{l}\beta \text {-catenin } \\
\text { localization }\end{array}$ & & & & 0.019 & & & 0.027 \\
\hline$\beta$-catenin $(\mathrm{m})$ & 59 & $8(13.6)$ & 1 (reference) & & $13(22.0)$ & 1 (reference) & \\
\hline$\beta$-catenin $(c)$ & 49 & $17(34.7)$ & $2.724(1.175-6.316)$ & & $21(42.9)$ & $2.182(1.092-4.360)$ & \\
\hline
\end{tabular}

RR, relative risk; 95\% CI, 95\% confidence interval; OS, overall survival; DFS, disease-free survival.

$(\mathrm{P}=0.021)$ and DFS $(\mathrm{P}=0.003)$ in patients with cyclophosphamide treatment (Fig. 6G and H). Combined ALDH1A1(high) and $\beta$-catenin(c) expression was associated with shorter OS $(\mathrm{P}=0.020)$ and DFS $(\mathrm{P}=0.012)$ in patients with cyclophosphamide treatment compared with other combined expression of ALDH1A1 and $\beta$-catenin (Fig. 6K and L).

The univariate analysis identified that the menopausal status, tumor size, histological stage, TNM stage, and lymph node metastasis were significantly associated with the OS and DFS in breast cancer patients (Table IV). In addition, ALDH1A1(high) expression was significantly associated with shorter OS of breast cancer patients. $\beta$-catenin(c) expression was significantly associated with shorter OS and DFS of breast cancer patients (Table IV). Furthermore, multivariate
Cox regression analysis found that histological grade was an independent prognostic factor for OS and DFS in breast cancer patients $(\mathrm{P}<0.05$, Table $\mathrm{V})$. Lymph node metastasis and histological grade were independent prognostic factors for shorter DFS in breast cancer patients $(\mathrm{P}<0.05$, Table $\mathrm{V})$.

\section{Discussion}

In this study, we performed tissue microarray-based immunohistochemistry to examine the expression of ALDH1A1 and $\beta$-catenin in 276 breast cancer patients. We found that ALDH1A1 overexpression was associated with lymph node metastasis and shorter OS in breast cancer patients. In addition, we found that the $\beta$-catenin(c) expression was associated 
Table V. Multivariate Cox regression analysis of the association between clinicopathological data and OS and DFS in breast cancer patients.

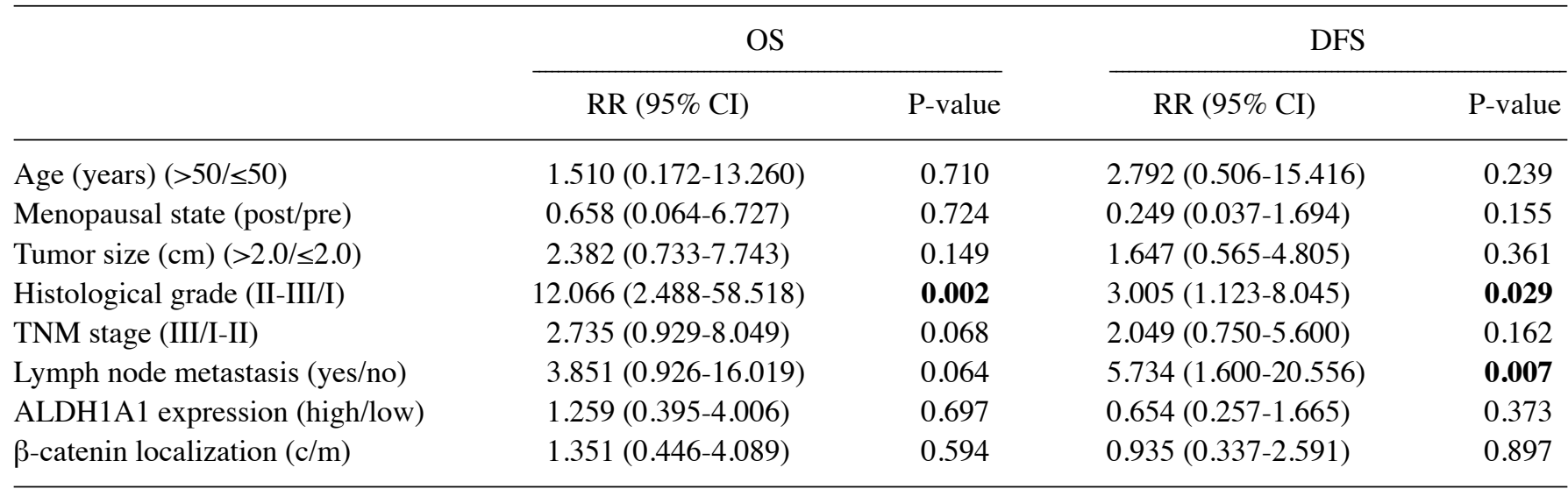

RR, relative risk; 95\% CI, 95\% confidence interval; OS, overall survival; DFS, disease-free survival.

with lymph node metastasis and shorter OS and DFS in breast cancer patients. Furthermore, combined expression of ALDH1A1(high) and $\beta$-catenin(c) was associated with shorter OS and DFS in breast cancer patients, especially in patients with cyclophosphamide treatment. Our findings suggest that translocation of $\beta$-catenin from the membrane to the cytoplasm may subsequently promote the expression of ALDH1A1 in breast cancer, and $\beta$-catenin-regulated ALDH1A1 may contribute to poor outcome of breast cancer patients.

In the present study, we examined the association of ALDH1A1 expression withclinicalcharacteristics and outcomes of breast cancer patients. We found that ALDH1A1(high) expression was associated with poor clinical outcome of breast cancer patients in agreement with previous studies showing that ALDH1A1 is associated with poor prognosis of breast cancer patients $(11,12)$. In addition, we found that ALDH1A1(high) expression was associated with lymph node metastasis, and patients with ALDH1A1(high) expression had more metastatic lymph nodes than those with ALDH1A1(low) expression. Consistent with our results, Khoury et al found that ALDH1A1 expression correlated with lymph node metastasis in breast cancer patients (24). Several studies have demonstrated that ALDH1 expression is associated with lymph node metastasis and poor survival in various tumors such as esophageal squamous cell carcinoma, gastric cancer, and rectal cancer (25-27). Our findings that ALDH1A1(high) was associated with lymph node metastasis and poor clinical outcome suggests that ALDH1A1 expression may define breast cancer with strong metastatic potential and be used for predicting disease outcome. Furthermore, ALDH1A1 is known to inactivate cyclophosphamide, and thus contribute to resistance to cyclophosphamide and poor clinical outcome of breast cancer patient $(14,24)$. Moreover, we found that ALDH1A1(high) expression was associated with shorter OS and DFS in patients with cyclophosphamide treatment, but not in those with non-cyclophosphamide treatment. Therefore, ALDH1A1 may be used as a biomarker to predict poor clinical outcome after cyclophosphamide treatment.

The Wnt signaling pathway is known to be involved in the development of breast cancer (28). During Wnt signaling activation, $\beta$-catenin is translocated from the membrane to the cytoplasm and the nucleus, where it promotes transcription of multiple protumorigenic genes via interaction with LEF/TCF transcription factors (20). The cytoplasmic accumulation of $\beta$-catenin has been found to be associated with poor clinical outcome of breast cancer patients $(20,29-32)$. In the present study, we found that the cytoplasmic expression of $\beta$-catenin was associated with grade II-II tumors, lymph node metastasis, and shorter OS and DFS in breast cancer patients. Our study supports that the cytoplasmic expression of $\beta$-catenin can be used as a prognostic marker for poor outcome of breast cancer patients. Although the mechanisms by which $\beta$-catenin is translocated from the membrane to the cytoplasm in breast cancer remain largely unclear, Adenomatous polyposis coli (APC), a scaffolding protein that regulate the cellular level of $\beta$-catenin in the Wnt signaling pathway (33), may be involved. It has been reported that the low expression of APC is associated with overexpression of $\beta$-catenin in the cytoplasm of breast cancer cells (34). In breast cancer cell lines, APC truncation leads to an increase in the cytoplasmic expression of $\beta$-catenin (35).

Although ALDH1A1 is known to be a CSC marker that is associated with poor prognosis of many tumors including breast cancer (10-12), little is known about the regulation of ALDH1A1 expression. Recently, it has been reported that ALDH1A1 is a direct target of $\beta$-catenin in ovarian cancer spheroids (15). In the present study, we found that $\beta$-catenin(c) expression occurred more frequently in ALDH1A1(high) breast cancer, and the expression level of ALDH1A was higher in $\beta$-catenin(c) breast cancer, suggesting that $\beta$-catenin may regulate the expression of ALDH1A1 in breast cancer. This idea is supported by the finding that the ALDH activity was reduced by the inhibition of Wnt/ $\beta$-catenin signaling by a TCF4-dominant negative construct in human breast cancer cells (36). Thus, $\beta$-catenin may regulate the expression of ALDH1A1 via interaction with the TCF4 transcription factor in breast cancer. In addition, we found that tumors with ALDH1A1(high) and $\beta$-catenin(c) expression were associated with lymph node metastasis and poor clinical outcome in 
breast cancer patients. Consistent with our findings, $\mathrm{Xu}$ et al reported that in patients with colorectal carcinoma, $\beta$-catenin was preferentially expressed in tumors with high expression of ALDH1A1, and were associated with high potential of metastasis and poor outcome (37). Furthermore, we found that ALDH1A1(high) and $\beta$-catenin(c) expression was associated with shorter OS and DFS in patients with cyclophosphamide treatment, suggesting that tumors with ALDH1A1(high) and $\beta$-catenin(c) expression exhibited resistance to cyclophosphamide treatment. ALDH1A1(high) and $\beta$-catenin(c) expression may be used for predicting poor outcome of breast cancer patients.

In summary, we investigated the expression of ALDH1A1 and $\beta$-catenin in breast cancer patients, and analyzed the association of their expression alone and in combination with the clinicopathological characteristics and prognosis of breast cancer patients. We found that ALDH1A1(high) and $\beta$-catenin(c) expression was associated with lymph node metastasis and worse clinical outcome in breast cancer patients, especially those receiving cyclophosphamide treatment. Combined expression of ALDH1A1(high) and $\beta$-catenin(c) was associated with lymph node metastasis, poor outcome, and resistance to cyclophosphamide treatment. Our study suggests that $\beta$-catenin may regulate ALDH1A1 expression in a subtype of breast cancer with ALDH1A1(high) and $\beta$-catenin(c) expression. ALDH1A1(high) and $\beta$-catenin(c) expression may be used as a biomarker for predicting poor prognosis in breast cancer following cyclophosphamide treatment.

\section{Acknowledgements}

This study was supported by grants from the National Natural Science Foundation of the People's Republic of China (no. 81373427), Program for Liaoning Innovative Research Team in University, LNIRT (LT2014016) and the Shenyang Science and Technology Plan Project (F14-232-6-05).

\section{References}

1. Stebbing $\mathbf{J}$ and Ellis P: An overview of drug development for metastatic breast cancer. Br J Nurs 21 (Suppl 4): S18-S22, 2012.

2. Li X, Lewis MT, Huang J, Gutierrez C, Osborne CK, Wu MF, Hilsenbeck SG, Pavlick A, Zhang X, Chamness GC, et al: Intrinsic resistance of tumorigenic breast cancer cells to chemotherapy. J Natl Cancer Inst 100: 672-679, 2008.

3. Sampieri K and Fodde R: Cancer stem cells and metastasis. Semin Cancer Biol 22: 187-193, 2012.

4. Neumeister V, Agarwal S, Bordeaux J, Camp RL and Rimm DL: In situ identification of putative cancer stem cells by multiplexing ALDH1, CD44, and cytokeratin identifies breast cancer patients with poor prognosis. Am J Pathol 176: 2131-2138, 2010.

5. Jiang F, Qiu Q, Khanna A, Todd NW, Deepak J, Xing L, Wang H, Liu Z, Su Y, Stass SA, et al: Aldehyde dehydrogenase 1 is a tumor stem cell-associated marker in lung cancer. Mol Cancer Res 7: 330-338, 2009.

6. Marhaba R, Klingbeil P, Nuebel T, Nazarenko I, Buechler MW and Zoeller M: CD44 and EpCAM: Cancer-initiating cell markers. Curr Mol Med 8: 784-804, 2008.

7. Nomura M, Fukuda T, Fujii K, Kawamura T, Tojo H, Kihara M, Bando Y, Gazdar AF, Tsuboi M, Oshiro H, et al: Preferential expression of potential markers for cancer stem cells in large cell neuroendocrine carcinoma of the lung. An FFPE proteomic study. J Clin Bioinforma 1: 23, 2011.

8. Feng D, Wang N,Hu J and Li W: Surface markers of hepatocellular cancer stem cells and their clinical potential. Neoplasma 61: 505-513, 2014.
9. Croker AK, Goodale D, Chu J, Postenka C, Hedley BD, Hess DA and Allan AL: High aldehyde dehydrogenase and expression of cancer stem cell markers selects for breast cancer cells with enhanced malignant and metastatic ability. J Cell Mol Med 13: 2236-2252, 2009.

10. Ginestier C, Hur MH, Charafe-Jauffret E, Monville F, Dutcher J, Brown M, Jacquemier J, Viens P, Kleer CG, Liu S, et al: ALDH1 is a marker of normal and malignant human mammary stem cells and a predictor of poor clinical outcome. Cell Stem Cell 1: 555-567, 2007.

11. Liu Y, Lv DL, Duan JJ, Xu SL, Zhang JF, Yang XJ, Zhang X, Cui YH, Bian XW and Yu SC: ALDH1A1 expression correlates with clinicopathologic features and poor prognosis of breast cancer patients: A systematic review and meta-analysis. BMC Cancer 14: 444, 2014.

12. Wu S, Xue W, Huang X, Yu X, Luo M, Huang Y, Liu Y, Bi Z, Qiu X and Bai S: Distinct prognostic values of ALDH1 isoenzymes in breast cancer. Tumour Biol 36: 2421-2426, 2015.

13. Pandrangi SL, Chikati R, Chauhan PS, Kumar CS, Banarji A and Saxena S: Effects of ellipticine on ALDH1A1-expressing breast cancer stem cells - an in vitro and in silico study. Tumour Biol 35: 723-737, 2014.

14. Sládek NE, Kollander R, Sreerama L and Kiang DT: Cellular levels of aldehyde dehydrogenases (ALDH1A1 and ALDH3A1) as predictors of therapeutic responses to cyclophosphamide-based chemotherapy of breast cancer: A retrospective study. Rational individualization of oxazaphosphorine-based cancer chemotherapeutic regimens. Cancer Chemother Pharmacol 49: 309-321, 2002.

15. Condello S, Morgan CA, Nagdas S, Cao L, Turek J, Hurley TD and Matei D: $\beta$-catenin-regulated ALDH1A1 is a target in ovarian cancer spheroids. Oncogene 34: 2297-2308, 2015.

16. Jiang W and Hiscox S: $\beta$-catenin - cell adhesion and beyond (Review). Int J Oncol 11: 635-641, 1997.

17. Madjd Z, Gheytanchi E, Erfani E and Asadi-Lari M: Application of stem cells in targeted therapy of breast cancer: A systematic review. Asian Pac J Cancer Prev 14: 2789-2800, 2013.

18. Lindvall $\mathrm{C}, \mathrm{Bu} \mathrm{W}$, Williams $\mathrm{BO}$ and $\mathrm{Li} \mathrm{Y}$ : Wnt signaling, stem cells, and the cellular origin of breast cancer. Stem Cell Rev 3: 157-168, 2007.

19. Arend RC, Londoño-Joshi AI, Straughn JM Jr and Buchsbaum DJ: The Wnt/ $\beta$-catenin pathway in ovarian cancer: A review. Gynecol Oncol 131: 772-779, 2013.

20. Lin SY, Xia W, Wang JC, Kwong KY, Spohn B, Wen Y, Pestell RG and Hung MC: Beta-catenin, a novel prognostic marker for breast cancer: Its roles in cyclin D1 expression and cancer progression. Proc Natl Acad Sci USA 97: 4262-4266, 2000.

21. Yu Z, Xiao Q, Zhao L, Ren J, Bai X, Sun M, Wu H, Liu X, Song Z, Yan Y, et al: DNA methyltransferase 1/3a overexpression in sporadic breast cancer is associated with reduced expression of estrogen receptor-alpha/breast cancer susceptibility gene 1 and poor prognosis. Mol Carcinog: Jan 25, 2014 (Epub ahead of print). doi: $10.1002 / \mathrm{mc} .22133$.

22. Fang Y, Wei J, Cao J, Zhao H, Liao B, Qiu S, Wang D, Luo J and Chen W: Protein expression of ZEB2 in renal cell carcinoma and its prognostic significance in patient survival. PLoS One 8: e62558, 2013

23. Zhu W, Cai MY, Tong ZT, Dong SS, Mai SJ, Liao YJ, Bian XW, Lin MC, Kung HF, Zeng YX, et al: Overexpression of EIF5A2 promotes colorectal carcinoma cell aggressiveness by upregulating MTA1 through C-myc to induce epithelial-mesenchymaltransition. Gut 61: 562-575, 2012.

24. Khoury T, Ademuyiwa FO, Chandrasekhar R, Jabbour M, Deleo A, Ferrone S, Wang Y and Wang X: Aldehyde dehydrogenase 1A1 expression in breast cancer is associated with stage, triple negativity, and outcome to neoadjuvant chemotherapy. Mod Pathol 25: 388-397, 2012.

25. Teng HW, Yang SH, Lin JK, Chen WS, Lin TC, Jiang JK, Yen CC, $\mathrm{Li} \mathrm{AF}$, Chen PC, Lan YT, et al: CIP2A is a predictor of poor prognosis in colon cancer. J Gastrointest Surg 16: 1037-1047, 2012.

26. Wakamatsu Y, Sakamoto N, Oo HZ, Naito Y, Uraoka N, Anami K, Sentani K, Oue N and Yasui W: Expression of cancer stem cell markers ALDH1, CD44 and CD133 in primary tumor and lymph node metastasis of gastric cancer. Pathol Int 62: 112-119, 2012.

27. Avoranta ST, Korkeila EA, Ristamäki RH, Syrjänen KJ, Carpén OM, Pyrhönen SO and Sundström JT: ALDH1 expression indicates chemotherapy resistance and poor outcome in node-negative rectal cancer. Hum Pathol 44: 966-974, 2013.

28. Howe LR and Brown AM: Wnt signaling and breast cancer. Cancer Biol Ther 3: 36-41, 2004. 
29. López-Knowles E, Zardawi SJ, McNeil CM, Millar EK, Crea P, Musgrove EA, Sutherland RL and O'Toole SA: Cytoplasmic localization of beta-catenin is a marker of poor outcome in breast cancer patients. Cancer Epidemiol Biomarkers Prev 19: 301-309, 2010.

30. Dolled-Filhart M, McCabe A, Giltnane J, Cregger M, Camp RL and Rimm DL: Quantitative in situ analysis of beta-catenin expression in breast cancer shows decreased expression is associated with poor outcome. Cancer Res 66: 5487-5494, 2006.

31. Geyer FC, Lacroix-Triki M, Savage K, Arnedos M, Lambros MB, MacKay A, Natrajan R and Reis-Filho JS: $\beta$-catenin pathway activation in breast cancer is associated with triple-negative phenotype but not with CTNNB1 mutation. Mod Pathol 24: 209-231, 2011.

32. Wang S, Li W, Lv S, Wang Y, Liu Z, Zhang J, Liu T and Niu Y: Abnormal expression of Nek 2 and $\beta$-catenin in breast carcinoma: Clinicopathological correlations. Histopathology 59: 631-642, 2011.

33. Barker N: The canonical Wnt/beta-catenin signalling pathway. Methods Mol Biol 468: 5-15, 2008.
34. Ozaki S, Ikeda S, Ishizaki Y, Kurihara T, Tokumoto N, Iseki M, Arihiro K, Kataoka T, Okajima M and Asahara T: Alterations and correlations of the components in the Wnt signaling pathway and its target genes in breast cancer. Oncol Rep 14: 1437-1443, 2005.

35. Schlosshauer PW, Brown SA, Eisinger K, Yan Q, Guglielminetti ER, Parsons R, Ellenson LH and Kitajewski J: APC truncation and increased beta-catenin levels in a human breast cancer cell line. Carcinogenesis 21: 1453-1456, 2000.

36. Debeb BG, Lacerda L, Xu W, Larson R, Solley T, Atkinson R, Sulman EP, Ueno NT, Krishnamurthy S, Reuben JM, et al: Histone deacetylase inhibitors stimulate dedifferentiation of human breast cancer cells through WNT/ $\beta$-catenin signaling. Stem Cells 30: 2366-2377, 2012.

37. Xu SL, Zeng DZ, Dong WG, Ding YQ, Rao J, Duan JJ, Liu Q, Yang J, Zhan N, Liu Y, et al: Distinct patterns of ALDH1A1 expression predict metastasis and poor outcome of colorectal carcinoma. Int J Clin Exp Pathol 7: 2976-2986, 2014. 